\title{
Frequency of and trends in fragrance allergy over a 15-year period
}

\author{
Andrea Nardelli ${ }^{1}$, An Carbonez ${ }^{2}$, Winfried Ottoy $^{2}$, Jacques Drieghe $^{1}$ and An Goossens ${ }^{1}$ \\ ${ }^{1}$ Department of Dermatology, University Hospital, Katholieke Universiteit Leuven, Leuven, Belgium and \\ ${ }^{2}$ University Centre for Statistics, Katholieke Universiteit Leuven, Heverlee, Belgium
}

Background: The widespread use of fragrance-containing products is probably the most important reason for its high impact in allergic contact dermatitis.

\begin{abstract}
Objectives: To describe the frequency of contact allergy to fragrance allergens as tested in the standard series, in relation to age, sex and lesion locations. To determine trends in frequency over the years and to study the association between positive tests observed with the different fragrance-allergy markers as well as between specific fragrance allergens and locations of the lesions.
\end{abstract}

Patients/Methods: 10128 patients underwent patch testing between January 1990 and December 2005 at the Dermatology department in Leuven.

Results: $1463(14.5 \%)$, that is, $380(26 \%)$ males and $1083(74 \%)$ females, reacted positively to at least 1 fragrance-allergy marker in the standard series: $9 \%$ to fragrance mix I, $6 \%$ to Myroxylon pereirae, and $4.8 \%$ to colophonium (often in association), $2.1 \%$ to hydroxyisohexyl 3-cyclohexene carboxaldehyde and $2.1 \%$ to fragrance mix II, the latter 2 allergens having been introduced more recently. Over the years, fragrance contact allergy has shown a fluctuating trend. Hands and face were the most commonly affected body sites. Moreover, a significant association was found between specific fragrance allergens and certain locations.

Conclusions: This study illustrates that fragrance contact allergy is common in patients suffering from contact dermatitis.

Key words: age; allergic contact dermatitis; epidemiology; fragrances; frequency; localization; odd ratio; relative risk; sex; trends. (C) Blackwell Munksgaard, 2008.

Accepted for publication 29 August 2007

\section{Introduction}

Adverse reactions to fragrances in perfumes and cosmetic products include allergic contact dermatitis (ACD), irritant contact dermatitis, photosensitivity, immediate reactions (contact urticaria), pigmented contact dermatitis, and (worsening of) respiratory problems (1).

Allergic contact dermatitis from fragrances results from a combination of repeated environmental exposure and age-related susceptibility factors (2) and is commonly observed in patch-test populations. Allergenic fragrance chemicals are indeed ubiquitous in our environment, not only in fine fragrances but also in a wide range of cosmetic, household, and industrial products (3) and even topical medicaments. Actually, together with preservative agents, they are the most important sensitizing culprits in cosmetic products $(4,5)$, with a frequency that is estimated to be around $1.5 \%$ in the general population (Denmark, $1.1 \%$ and Norway, $1.8 \%)(6,7)$ and between $6-14 \%$ in contact dermatitis patients (8).

The main tool for diagnosing contact allergy to fragrances is the European standard patch-test series. This standard series consists of (i) fragrance mix I (FM I), a mixture of 1 natural compound, oak moss absolute (Evernia prunastri), and the synthetic fragrances isoeugenol, hydroxycitronellal, cinnamal, cinnamic alcohol, eugenol, $\alpha$-amyl cinnamal, and geraniol, at a concentration of $1 \%$ each, (ii) Myroxylon pereirae (MP or balsam of Peru), and to a less extend also (iii) colophonium. New 
screening substances have emerged from multicenter studies in order to increase the ability to diagnose fragrance allergy $(9,10)$. As a result of this, since 2005, a mixture of 6 additional fragrance materials has been commercialized for future introduction into the standard series, that is, fragrance mix II (FM II) that contains hydroxyisohexyl 3-cyclohexene carboxaldehyde (HICC or Lyral ${ }^{\mathbb{R}}$ ), citral, farnesol, coumarin, citronellol, and $\alpha$-hexyl cinnamal.

The aims of this retrospective study were as follows:

(1) To describe the frequency of contact allergy to fragrance allergens as tested in the standard series.

(2) To determine trends in frequency over the years.

(3) To characterize the age, sex, and lesion location of the fragrance-allergic patients.

(4) To study the association between the positive tests observed with the different fragrance-allergy markers.

(5) To study the association between specific fragrance allergens and localization of the dermatitis.

\section{Materials and Methods}

From January 1990 until December 2005, 10128 consecutive patients presenting with eczematous dermatitis were patch tested in the Contact Allergy Unit of the Katholieke Universiteit Leuven with a European Standard series (Hermal, Reinbeck, Germany) and (when indicated) also with other allergens.

The patch tests were administered with Van Der Bend patch-test chambers (Van Der Bend, Brielle, the Netherlands) applied on the back with Micropore $^{\mathrm{TM}}$ (3M Health Care, Borken, Germany) and fixed with Fixomull ${ }^{\mathrm{B}}$ (Beiersdorf, Germany) and later on with Mefix ${ }^{\circledR}$ (Mölnlycke Health Care, Göteborg, Sweden) as adhesive tapes. The patch-tested readings were performed according to the international guidelines by the International Contact Dermatitis Research Group (11) after 2 days, 3 days (exceptionally) and 4 days and sometimes later.

All data were retrieved from and evaluated with a patient database developed in our department $(12,13)$. The fragrance-related allergens present in the standard series were studied in an explorative way. In the study of associations between patchtest results and the location of the lesions, the data were presented in a $2 \times 2$ contingency table. As an appropriate statistical measure to compute the strength and the direction of the association, we used the odds ratio (OR), expressing the occurrence of positive reactions in 1 group as compared with another group, and/or the relative risk, and their corresponding 95\% confidence interval (CI). If the $\mathrm{CI}$ is different from the value 1 , there is a significant association between the row and the column variables. The statistical analysis in both the male and the female populations was performed by using the SAS SOFTWARE SYSTEM version 9.1 (SAS Institute Inc., Cary, NC, USA).

\section{Results}

Among the entire population of 10128 patients patch tested, 3491 were males $(34.5 \%)$ and 6637 were females $(65.5 \%) .5690(56 \%)$ presented with a contact-allergic reaction to at least 1 of the substances tested, and $1463(14.5 \%)$, that is, 380 $(26 \%)$ males and $1083(74 \%)$ females, reacted positively to at least 1 fragrance marker, making up $26 \%$ of those with contact allergy.

The OR for females is $0.19 \quad\left(\mathrm{OR}_{\mathrm{f}}=1083 /\right.$ $5554=0.1950)$, expressing the probability to have a positive reaction to be 0.19 higher than the probability of having a negative reaction to fragrance. The odds for males is only 0.12 (odds $\left._{\mathrm{m}}=380 / 3111=0.1221\right)$. The OR is then 1.6 with a $95 \%$ CI $(1.41-1.81)$ or the probability of having a positive patch-test reaction in females being 1.6 times larger than in males.

The mean age in fragrance-allergic patients was 45 for males and 44 for females (both with \pm 17 SD), compared with 39 years both for males and females $( \pm 17$ SD) in the non-fragrance allergic patients $(n=8665)$. For a more convenient handling of the data, the age of the patients was divided into groups with a span of 20 years (Fig. 1). The frequency of fragrance allergy gradually increased from young to adult age, the highest peak between 20 and 40 years $(40 \%)$ for females and between 40 and 60 years $(37.6 \%)$ for males, to decrease gradually again later on.

In the whole group, there were 3626 atopic patients $(36 \%)$ (atopic dermatitis, asthma and/or allergic rhinitis), 521 of whom (14.4\%) presented with a positive reaction to at least 1 fragranceallergy marker, compared with $942(14.5 \%)$ in the 6502 non-atopic patients $(64 \%)$. Thus, positive reactions to a fragrance-allergy marker and atopy were not significantly associated.

As regarding the fragrance-allergy markers, 9\% of the population tested $(n=924)$ presented with a positive reaction to FM I, $6 \%(n=617)$ to MP, $4.8 \%(n=489)$ to colophonium (Table 1$), 2.1 \%$ ( $n=62$ in 2901 patients tested) to HICC, and $2.1 \%$ ( $n=7$ in 335 patients tested) to FM II, the latter allergens being introduced more 

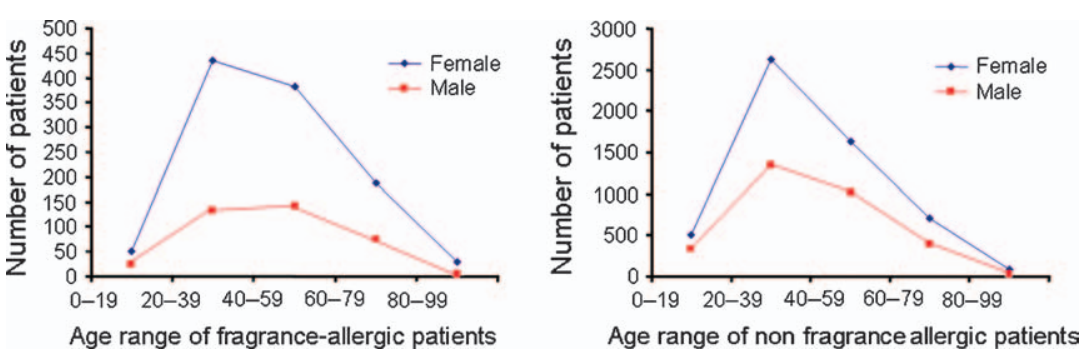

Fig. 1. Distribution of age of fragrance(right panel) and non-fragrance allergic patients (left panel).
Table 1. Positive reactions observed to FM I, MP and C

\begin{tabular}{llll}
\hline Markers & $\begin{array}{l}\text { Total } \\
(n=10 ~ 128), n(\%)\end{array}$ & $\begin{array}{l}\text { Male } \\
(n=1463)\end{array}$ & $\begin{array}{l}\text { Female } \\
(n=6637)\end{array}$ \\
\hline FM I & $924(9.12)$ & 258 & 666 \\
MP & $617(6.09)$ & 162 & 455 \\
C & $489(4.83)$ & 113 & 376 \\
\hline
\end{tabular}

C, colophonium; FM I, fragrance mix I; MP, Myroxylon pereirae.

recently, in 2002 and 2005, respectively. 30 patients out of $62(48.4 \%)$ reacted both to FM I and to HICC. The total number of positive reactions observed is, of course, higher than the number of patients as many of them suffered from multiple sensitivities. Among those reacting to FM I, MP, or colophonium, females represented $72 \%, 74 \%$, and $77 \%$ and the males $28 \%, 26 \%$, and $23 \%$, respectively.

The results of the positive patch-test reactions obtained with FM I, MP, and colophonium over the years are given in Table 2, which is illustrated in Fig. 2.

The associated positive tests observed with the different fragrance-allergy markers are given in Table 3 (multiple hypotheses testing for all the different fragrance-allergy markers were taken into account), showing a strong association for all comparisons reported. When the associations were significant, the OR was computed. As an example, an OR of 11 (Table 3) means that the odds of a positive reaction to colophonium is 11 times higher for patients with a positive reaction to FM I than for non-FM I allergic patients.

The localization of the lesions in the fragrance $(n=1463)$ and non-fragrance $(n=8665)$ allergic patients are visualized in Fig. 3. Hands $(31.2 \%)$, face $(27.2 \%)$, lower legs $(16.6 \%)$, and feet $(15.3 \%)$ were the most commonly affected body sites in fragrance-sensitive individuals, particularly in females. Significant associations were found for arms (relative risk, 1.24; 95\% CI, 1.09-1.41), lower legs (relative risk, $1.71 ; 95 \% \mathrm{CI}, 1.51-1.93$ ) and upper legs (relative risk, 10.82; 95\% CI, 8.5613.68), and axillae (relative risk, 1.51; 95\% CI, $1.21-1.90$ ). For hands (relative risk, $0.8 ; 95 \% \mathrm{CI}$ 0.76-0.93), there is a negative correlation with fragrance allergy though.

Table 4 shows the results of the association between localization and specific fragrance allergens. Of 40 combinations of localization by marker, 9 were significantly associated either for males or for females, or both. As this was an explorative study, a correction for multiple comparisons was not used.

\section{Discussion}

It is well known that after nickel, contact allergy to fragrance components is the most common finding among contact-allergy patients $(14,15)$. This was also true in our female population studied and, since 1995 to 2006, FM I and pphenylenediamine (data not shown) were, in alternation, the 2 most frequent allergens in our male population. The majority of patients with fragrance sensitivity were women (16-18), which reflects a greater exposure to fragranced cosmetics in this population. However, in view of an increasing usage also in men, the sex difference may become less prominent in the future.

Buckley et al. argued that ACD from fragrances has an age-related susceptibility (2) and observed a peak in the 60s for females and in the 70 s for males (2). The highest peak observed between the ages 20 and 40 in our female population could be explained by the greater personal use of

Table 2. Percentage of positive patch-test results obtained with fragrance mix I, Myroxylon pereirae and colophonium over the years

\begin{tabular}{|c|c|c|c|c|c|c|c|c|c|c|c|c|c|c|c|c|}
\hline & 1990 & 1991 & 1992 & 1993 & 1994 & 1995 & 1996 & 1997 & 1998 & 1999 & 2000 & 2001 & 2002 & 2003 & 2004 & 2005 \\
\hline FM I & 7.2 & 7.3 & 6.6 & 5.6 & 13.1 & 9.6 & 10.1 & 8.1 & 11.9 & 13.9 & 12.5 & 9.7 & 8.4 & 8.3 & 9.3 & 7.7 \\
\hline MP & 6.5 & 6.8 & 5.2 & 4.8 & 4.8 & 6.5 & 6.7 & 5.6 & 6.1 & 6.8 & 7.7 & 6.9 & 6.6 & 6.3 & 7.9 & 5.0 \\
\hline $\mathrm{C}$ & 5.5 & 5.5 & 3.7 & 3.8 & 6.1 & 4.8 & 4.7 & 3.7 & 6.3 & 5.7 & 5.6 & 5.4 & 4.6 & 4.3 & 5.3 & 4.3 \\
\hline
\end{tabular}




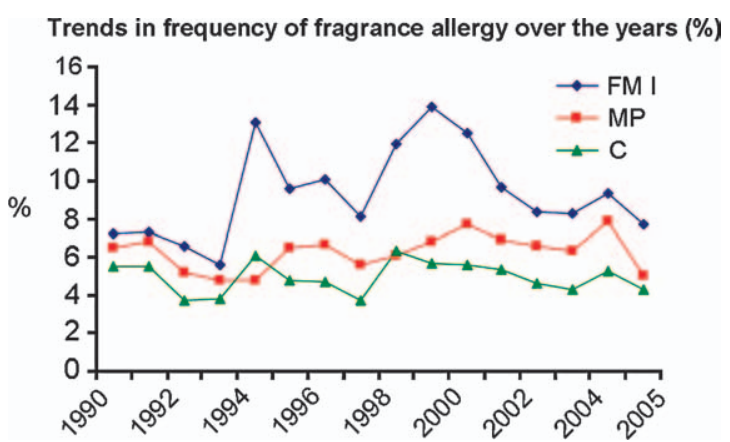

Fig. 2. Evolution of the relative frequency over the years of positive reactions to fragrance-allergy markers. C, Colophonium; FM I, fragrance mix I; MP, Myroxylon pereirae.

Table 3. Odds ratios and corresponding 95\% CIs for the different fragrance-allergy markers

\begin{tabular}{llc}
\hline & $\begin{array}{l}\text { Males } \\
\text { Ollergens }\end{array}$ & $\begin{array}{l}\text { Females } \\
\text { OR }(95 \% \mathrm{CI})\end{array}$ \\
\hline FM I and C & $11.14(7.48-16.59)$ & $5.11(4.05-6.46)$ \\
FM I and MP & $35.74(24.98-51.15)$ & $18.96(15.35-23.42)$ \\
FM I and HICC & $25.63(6.37-103.10)$ & $17.50(10.09-30.34)$ \\
C and MP & $5.95(3.65-9.70)$ & $5.35(4.14-6.92)$ \\
\hline
\end{tabular}

C, colophonium; CI, confidence interval; FM I, fragrance mix I; HICC, hydroxyl-isohexyl cyclohexene carboxaldehyde;

MP, Myroxylon pereirae; OR, odds ratio.

fragranced products in young women in particular. In males, the highest peak was observed between the ages 40 and 60 . This could perhaps be explained by a different patient selection or a different consumer exposure in both centres.

With regard to the fragrance-allergy markers, FM I has been regarded as an adequate screening test for fragrance sensitivity, although it has been reported that as much as $33 \%$ of fragrance sensitivity may be missed (19), if it is used as the only test substance. This percentage is probably too low and depends on which fragrance chemicals or products the subjects are tested with. Indeed, the more fragrance allergens tested, the more positive reactions can be observed. In most epidemiological studies, as well in the current study, the patch-test result of patients reacting to their own perfume and deodorant, for example, or to any other fragrance allergen, have not been considered. Besides FM I, our results also take into consideration MP and colophonium, for which, in agreement with other studies, including a recent report from Austria (20), positive reactions are significantly associated in both sexes. Indeed, this can be explained by the fact that these products share similar components.

In fact, Hjorth has already reported in 1961 that gums and resins such as MP and benzoin are strong sensitizers, associated with fragrance allergy (21). He showed that the most important allergen of MP is formed by the polymerization of a 'protoresin' (monomer or low polymer), which is an ester of benzoic acid or cinnamic acid and coniferyl alcohol; however, many other allergenic components are present such as benzyl cinnamate, eugenol, methyl cinnamate, benzyl benzoate, vanillin, cinnamic acid, cinnamic alcohol, cinnamal, and benzyl salicylate (22), all present in or related to other allergenic fragrance materials. The crude MP, as such, has not been used in perfumery since 1982, when the International Fragrance Association banned its use in fragrances. Since 1995 (23, 24), MP has been incorporated in fragrances as an extract or distillate, but could be, according to a recent study, as allergenic as the crude product (M. Bruze, Malmö University Hospital, Lund University, Malmö, Sweden, personal communication).

Colophonium, originating from pine trees (25), often co-reacts with fragrance allergens; their major constituents are resin acids, that is, abietic acid and dehydroabietic acid, of which the oxidized derivatives are responsible for contact allergy (26). The same allergens have also been identified in the FM I component 'Evernia prunastri' or 'oak moss', either as contaminants or as ingredients of 'Evernia furfuracea' or 'tree moss', also derived from pine trees and often used by the perfume industry as a cheaper substitute for oak moss $(27,28)$. Besides oxidized terpenes, however, oak moss contains a large number of other ingredients, including atranalol and chloroatranalol, which are perhaps among the most allergenic substances ever identified (27), that is, still at
Fig. 3. Visualization of localization of the lesions in fragrance-allergic $(n=1463) \quad$ (right panel) and nonfragrance allergic patients $(n=8665)$ (left panel).
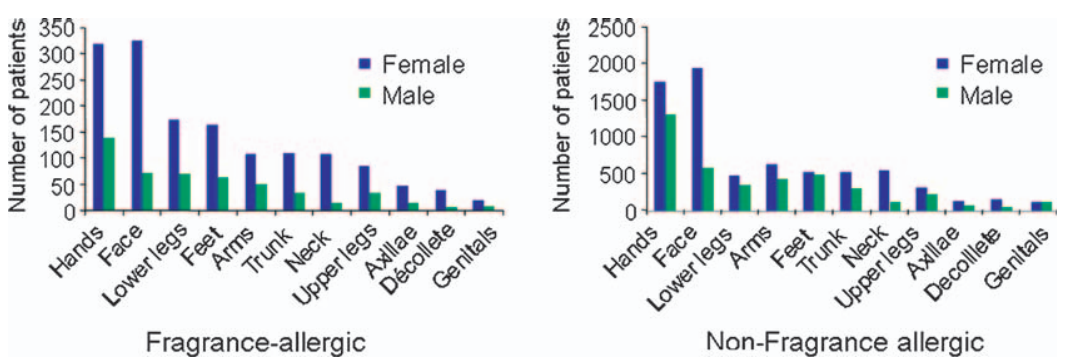
Table 4. Odds ratios and corresponding $95 \% \mathrm{CI}$ for the association between localizations and the fragrance allergens

\begin{tabular}{lcl}
\hline & Males & Females \\
Ollergens & OR $(95 \%$ CI $)$ & OR $(95 \%$ CI $)$ \\
\hline Axillae and FM I & $2.32(1.29-4.16)$ & $2.22(1.54-3.20)$ \\
Axillae and HICC & $4.65^{\mathrm{NS}}(0.57-37.63)^{\mathrm{NS}}$ & $7.28(3.62-14.62)$ \\
Trunk and HICC & $1.20^{\mathrm{NS}}(0.15-9.64)^{\mathrm{NS}}$ & $2.02(1.01-4.02)$ \\
Upper leg and C & $1.17^{\mathrm{NS}}(0.60-2.26)^{\mathrm{NS}}$ & $1.48(1.01-2.16)$ \\
Upper leg and MP & $1.23^{\mathrm{NS}}(0.71-2.13)^{\mathrm{NS}}$ & $1.50(1.06-2.12)$ \\
Lower leg and FM I & $2.17(1.58-2.99)$ & $1.94(1.55-2.43)$ \\
Lower leg and MP & $2.07(1.39-3.06)$ & $2.20(1.71-2.84)$ \\
Lower leg and C & $1.96(1.22-3.13)$ & $2.10(1.59-2.77)$ \\
Feet and C & $2.14(1.40-3.25)$ & $2.62(2.03-3.39)$ \\
\hline
\end{tabular}

C, Colophonium; CI, confidence interval; FM I, fragrance mix I; HICC, hydroxyl-isohexyl cyclohexene carboxaldehyde; MP, Myroxylon pereirae; NS, non-significant values; OR, odds ratio.

0.1 p.p.m. (the individual components of FM I will be discussed separately in a further study).

Besides FM I, MP, and colophonium, positive reactions to FM II, in which $\mathrm{HICC}$ is the most common sensitizer $(9,10)$, and HICC itself were also considered, although tested in a minority of the patients. The percentage of positive reactions found for HICC $(2.1 \%)$ is in agreement with the prevalence found in other European studies, that is, $1.5-3 \%(9,29-32)$. This is in contrast to North America where the prevalence was found to be only $0.4 \%$ (33), the difference being attributed to the presence of HICC in high concentrations in deodorants in the EU, likely to induce sensitization in occluded areas. Contact allergy to a fragrance would have been missed in 32 out of 62 $(52 \%)$ patients if we had only tested with FM I and not with HICC. This is much higher than what has been reported by Frosch et al., that is, in $7.2 \%$ of the patients $(29,30)$. Because of the low number of patient tested and reacting to FM II, we are not able to formulate any conclusion about missed reactions to this mixture. Frosch et al. previously reported that $1 / 3$ of the patients reacting to FM II were negative to FM I (10). Geier et al. in a large German study reported that 535 out of $6968(7.7 \%)$ patients reacted to FM I, while 321 of $6968(4.6 \%)$ were positive to FM II. 151 of them reacted to both allergens, representing $28 \%$ of those with contact allergy to FM I and $47 \%$ of those allergic to FM II. FM II has thus acceptable diagnostic qualities and is helpful as an additional tool in detecting fragrance allergy (34).

Concerning the trends in the fragrance sensitivity rate (Table 2 and Fig. 2), a fluctuating trend, either increasing or decreasing, was observed. For FM I, the frequency was highest from 1998 to 2000 and has then decreased in recent years. In 2006 (data not shown), the frequency was the same as in 2001, that is, 9.65. In the past decade, the prevalence of fragrance sensitivity, evaluated by testing only with FM I, varied considerably. A percentage between 7 and 10 was observed among patch-tested patients in Europe $(5,35-39)$. In Denmark, it rose from $4.1 \%$ in $1985-1986$ to $9.9 \%$ in 1997-1998 (40), and a similar rising tendency was reported in Slovenia as well (41). However, a recent decrease in FM I sensitivity has been reported in several studies $(5,19,20,35,36)$, for example, according to a recent European multicentre survey, the fragrance sensitivity rate has decreased significantly from $13.1 \%$ in 1999 to $7.8 \%$ in 2002 (42). This was also observed in the USA, where the fragrance sensitivity rate diminished from $14 \%$ to $11.4 \%$ in the late 1990 s $(8,43)$ and, according to a more recent study, even down to $5.9 \%$ (33). In the future, perhaps a further decrease in positive reactions to FM I allergens may be expected. Indeed, because of the labelling of 26 fragrance components by EU legislation (44), including those of FM I, as well as the outcome of recent dermatological studies on fragrance allergy and subsequent legal requirements [for example, reducing the concentration of isoeugenol (45)], the policy of some fragrance companies has changed. For instance, it has been shown that the new prestige perfumes contain less often or lesser amounts of fragrance chemicals included in FM I, compared with the older perfumes (46). Moreover, deodorants and domestic and occupational products quite often contain fragrance chemicals different from those present in FM I, such as limonene $(47,48)$. This underlines the usefulness of new screening substances for the purpose of increasing the ability to diagnose fragrance allergy $(9,10)$. In 2005, Hasan et al. have shown that in contrast to FM I, there is a significant increase in the sensitivity rate to MP in recent years (38), whereas the sensitivity rate to MP has been rather stable over the years in our study.

As to the lesion localization, according to our study, hands, face, lower legs, and feet are the most commonly affected body sites. Although fragrance allergy is not significantly associated with hand dermatitis, the hands are the most frequently involved sites in fragrance-allergic women (in men, hand dermatitis is more often because of other, more occupation-related allergens). This is not surprising (49-51) as many contactants do indeed contain fragrances: household products, cleaners, waxes, polishes, textile softeners, etc., and, of course, nearly all cosmetics (and many topical medications) are applied by the hands, sometimes also on the skin of other family members or pets (52). Moreover, patients may suffer first from irritant or atopic hand dermatitis, which may later be complicated by contact allergy to 
products used for treatment (fragranced topical drugs) or prevention (hand creams and lotions) or to other contacted perfumed products. Last but not least, dyshidrotic eruptions have been ascribed to ingestion of spices (53), which may contain fragrance chemicals or may cross-react with them (54).

As to the face, the sensitive facial skin and the eyelids in particular are susceptible to develop ACD from fragranced skin-care products, decorative cosmetics, and cleansing preparations and sometimes even from airborne exposure $(55,56)$. Moreover, microtraumata from shaving facilitate (photo) contact allergy to aftershave fragrances (57), but also other skin diseases such as atopic dermatitis and even facial psoriasis may be induced or aggravated by ACD from fragrances (58). In our population, although the face was a common localization, the association with fragrance allergy was not significant. Indeed, preservative agents, in particular, and, to a lower extend, emulsifiers are also important cosmetic allergens causing ACD on the face.

Significant associations between the neck, skin behind the ear or décolleté that are other usual sites of fragrance contact dermatitis were not observed. In these areas, nickel and cobalt present in cheap metal jewels are common allergens as well. The latter body sites as well as the axillae, notwithstanding its significant association with 'fragrance' allergy (Table 4), are certainly underrepresented in our study. Indeed, in general, the relation with the causal product being obvious (59, 60), patients consult because of dermatitis located elsewhere (which is indicated in our patient file as being the first site affected), and, after identification of fragrance allergy, they then often recall having previously suffered from skin reactions to toilet waters or deodorants, respectively. Reactions to FM I and to HICC (in females) were significantly associated with locations in the axillae, as has been reported earlier (61).

Contact allergy to FM I, colophonium, and also MP is often found in patients, especially females suffering from leg ulcers, who are exposed to fragrance-containing medications, adhesive tapes, etc. This will be investigated separately in a further study on iatrogenic dermatitis caused by fragrances. As to the feet, colophonium is certainly the main culprit in shoe dermatitis, not as a fragrance allergen, of course, but because it is used as a tackifier (32).

Significant, but perhaps unexpected associations between fragrance allergy and locations such as the trunk, arms, and also upper legs were found in females, although not with a specific fragrance chemical. This most probably reflects the use of fragrance-containing body creams and lotions and perhaps skin-cleansing products, in which, besides preservative agents, fragrances are the most important allergens. Furthermore, other existing eczematous conditions such as those located in the perianal or vulvar area (62) may also be complicated by fragrance allergy.

\section{Conclusions}

This study illustrates that also in our department, fragrance contact allergy is commonly observed in patients suffering from contact dermatitis, both in males and in females, and has shown a fluctuating trend over the years. Positive patch tests to the fragrance-allergy markers, that is, FM, M. pereirae (balsam of Peru), and colophonium in the standard series are frequently associated. The hands and face were the most commonly affected body sites, and significant associations were found for legs, arms, and axillae. Some fragrance allergens are specifically related to certain lesion localizations. Moreover, although HICC and FM II were only more recently introduced in the standard series, a significant association was found between HICC and axillae (in females).

However, not only the fragrance-allergy markers present or those being introduced in the standard series need to be taken into account, but also the products used by the patients, essential oils and other individual fragrance allergens as this certainly increases the detection of fragrance allergy (data to be published).

Special attention to safety assessments must be made for fragranced products and, particularly, for those intended for use on potentially traumatized skin, that is, hands, beard region, shaved legs, occluded areas or areas of high absorption, that is, eyelids, axillae, and genitals, or in chronic dermatitis, that is, stasis dermatitis/leg ulcer patients.

\section{Acknowledgement}

This epidemiological study on 'Fragrance allergy: frequency and trends' was partly supported by the 'Prix Jean Darier des laboratories SVR', Paris, December 2006.

\section{References}

1. de Groot A C. Dermatological problems linked to perfumes. In: Handbook of Cosmetic Science and Technology, Barel A O, Paye M, Maibach H I (eds): New York, Marcel Dekker, Inc, 2001: pp. 89-94.

2. Buckley D A, Rycroft R J, White I R, McFadden J P. The frequency of fragrance allergy in patch-tested patients increases with their age. $\mathrm{Br} J$ Dermatol 2003: 149: 986-989. 
3. Matura M, Goossens A, Bordalo O, Garcia-Bravo B, Magnusson K, Wrangsjo K, Karlberg A T. Patch testing with oxidized R- (+)-limonene and its hydroperoxide fraction. Contact Dermatitis 2003: 49: 15-21.

4. Goossens A. Sensitizing substances. In: Dry Skin and Moisturizers. Chemistry and Function, 2nd edition, Loden M, Maibach H I (eds): Boca Raton, Taylor \& Francis, 2006: pp. 515-522.

5. Schnuch A, Lessman H, Geier J, Frosch P J, Uter W. Contact allergy to fragrances: frequencies of sensitization from 1996 to 2002. Results of the IVDK. Contact Dermatitis 2004: 50: $65-76$.

6. Nielsen N H, Menne T. Allergic contact sensitization in an unselected Danish population. The Glostrup Allergy Study, Denmark. Acta Derm Venereol 1992: 72: 456-460.

7. Dotterud L K, Smith-Sivertsen T. Allergic contact sensitization in the general adult population: a population-based study from Northern Norway. Contact Dermatitis 2007: 56: $10-15$.

8. Marks J G, Belsito D V, DeLeo V A et al. North American Contact Dermatitis Group patch test results for the detection of delayed-type hypersensitivity to topical allergens. $J \mathrm{Am}$ Acad Dermatol 1998: 38: 911-918.

9. Frosch P J, Rastogi S C, Pirker C et al. Patch testing with a new fragrance mix-reactivity to the individual constituents and chemical detection in relevant cosmetic products. Contact Dermatitis 2005: 52: 216-225.

10. Frosch P J, Pirker C, Rastogi S C et al. Patch testing with a new fragrance mix detects additional patients sensitive to perfumes and missed by the current fragrance mix. Contact Dermatitis 2005: 52: 207-215.

11. Wahlberg J E. Patch testing. In: Textbook of Contact Dermatitis, 3rd edition, Rycroft R J G, Menné T, Frosch P J, Lepoittevin J P (eds): Berlin, Heidelberg, Springer, 2001: pp. 435-468.

12. Drieghe J, Goossens A. On-line internet facilities for contact allergy software: CDESK/PRO. Contact Dermatitis 2002: 47: 243 .

13. Goossens A, Drieghe J. Computer applications in contact allergy. Contact Dermatitis 1998: 38: 51-52.

14. de Groot A C, Frosch P J. Adverse reactions to fragrances. A clinical review. Contact Dermatitis 1997: 36: 57-86.

15. Nielsen $\mathrm{N} H$, Menne T. Nickel sensitization and ear piercing in an unselected Danish population. Glostrup Allergy Study. Contact Dermatitis 1993: 29: 16-21.

16. Scheinman P L. Allergic contact dermatitis to fragrance: a review. Am J Contact Dermat 1996: 7: 65-76.

17. Malanin $G$, Ohela K. Allergic reactions to fragrance-mix and its components. Contact Dermatitis 1989: 21: 62-63.

18. Malten K E, van Ketel W G, Nater J P, Liem D H. Reactions in selected patients to 22 fragrance materials. Contact Dermatitis 1984: 11: 1-10.

19. Larsen W, Nakayama H, Fischer T et al. A study of new fragrance mixtures. Am J Contact Dermat 1998: 9: 202-206.

20. Wohrl S, Hemmer W, Focke M, Gotz M, Jarisch R. The significance of fragrance mix, balsam of Peru, colophony and propolis as screening tools in the detection of fragrance allergy. Br J Dermatol 2001: 145: 268-273.

21. Hjorth N. Eczematous allergy to balsams, allied perfumes and flavouring agents, with special reference to balsam of Peru. Acta Derm Venereol 1961: 41: 1-216.

22. Larsen W G. Perfume dermatitis: a study of 20 patients. Arch Dermatol 1977: 113: 623-626.

23. Api A M. Only Peru balsam extracts or distillates are used in perfumery. Contact Dermatitis 2006: 54: 179.

24. Avalos-Peralta P, Garcia-Bravo B, Camacho F M. Sensitivity to Myroxylon pereirae resin (balsam of Peru). A study of 50 cases. Contact Dermatitis 2005: 52: 304-306.

25. Karlberg A T, Bohlinder K, Boman A, Hacksell U, Hermansson J, Jacobsson S, Nilsson J. Identification of 15-hydroperoxyabietic acid as a contact allergen to Portuguese colophony. J Pharm Pharmacol 1988: 40: 42-47.
26. Karlberg A T. Air oxidation increases the allergenic potential of tall oil rosin. Colophony contact allergens also identified in tall oil rosin. Am J Contact Dermat 1991: 2: 43-49.

27. Johansen J D, Heydorn S, Menne T. Oak moss extracts in the diagnosis of fragrance contact allergy. Contact Dermatitis 2002: 46: 157-161.

28. Lepoittevin J P, Meschkat E, Huygens S, Goosens A. Presence of resin acids in "Oakmoss" patch test material: a source of misdiagnosis? J Invest Dermatol 2000: 115: 129-130.

29. Frosch P J, Johansen J D, Menne T et al. Lyral is an important sensitizer in patients sensitive to fragrances. Br J Dermatol 1999: 141: 1076-1083.

30. Frosch P J, Johansen J D, Menne T et al. Further important sensitizers in patients sensitive to fragrances. Contact Dermatitis 2002: 47: 78-85.

31. Baxter K F, Wilkinson S M, Kirk S J. Hydroxymethyl pentylcyclohexene-carboxaldehyde (Lyral) as a fragrance allergen in the UK. Contact Dermatitis 2003: 48: 117-118.

32. Nardelli A, Degreef H, Goossens A. Contact allergic reactions of the vulva: a 14-year review. Dermatitis 2004: 15: 131-136.

33. Belsito D V, Fowler J F Jr, Sasseville D, Marks J G Jr, De Leo V A, Storrs F J. Delayed-type hypersensitivity to fragrance materials in a select North American population. Dermatitis 2006: 17: 23-28.

34. Geier J, Lessmann H, Uter W, Schnuch A. Experiences with fragrance mix II - the German perspective. Contact Dermatitis 2006: 55: 12 (Abstract)

35. Schnuch A, Geier J, Uter W et al. National rates and regional differences in sensitization to allergens of the standard series. Population-adjusted frequencies of sensitization (PAFS) in 40,000 patients from a multicenter study (IVDK). Contact Dermatitis 1997: 37: 200-209.

36. Temesvari E, Nemeth I, Balo-Banga M J et al. Multicentre study of fragrance allergy in Hungary. Immediate and late type reactions. Contact Dermatitis 2002: 46: 325-330.

37. Bangha E, Elsner P. Sensitizations to allergens of the European standard series at the Department of Dermatology in Zurich 1990-1994. Dermatology 1996: 193: 17-21.

38. Hasan T, Rantanen T, Alanko K et al. Patch test reactions to cosmetic allergens in 1995-1997 and 2000-2002 in Finland - a multicentre study. Contact Dermatitis 2005: 53: 40-45.

39. Buckley D A, Wakelin S H, Seed P T, Holloway D, Rycroft R J, White I R, McFadden J P. The frequency of fragrance allergy in a patch-test population over a 17 -year period. Br J Dermatol 2000: 142: 279-283.

40. Johansen J, Menne T, Christophersen J, Kaaber K, Veien N. Changes in the pattern of sensitization to common contact allergens in Denmark between 1985-86 and 1997-98, with a special view to the effect of preventive strategies. $\mathrm{Br} J$ Dermatol 2000: 142: 490-495.

41. Lunder T, Kansky A. Increase in contact allergy to fragrances: patch-test results 1989-1998. Contact Dermatitis 2000: 43: 107-109.

42. Bruynzeel D P, Diepgen T L, Andersen K E et al. European Environmental and Contact Dermatitis Research Group. Monitoring the European standard series in 10 centres 1996-2000. Contact Dermatitis 2005: 53: 146-149.

43. Marks J G Jr, Belsito D V, DeLeo V A et al. North American Contact Dermatitis Group patch-test results, 1996-1998. Arch Dermatol 2000: 136: 272-273.

44. EU Directive 2003/15/EC of the European Parliament and of the Council of the 27 February 2003 amending Council Directive 76/768/EEC on the approximation of the laws of the Member States relating to cosmetic products (7th Amendment of the European Cosmetic Directive). Official $J$ Eur Union 2003: L66: 26-35.

45. Tanaka S, Royds C, Buckley D et al. Contact allergy to isoeugenol and its derivatives: problems with allergen substitution. Contact Dermatitis 2004: 51: 288-291. 
46. Rastogi S C, Menne T, Johansen J D. The composition of fine fragrances is changing. Contact Dermatitis 2003: 48: $130-132$.

47. Rastogi S C, Johansen J D, Frosch P et al. Deodorants on the European market: quantitative chemical analysis of 21 fragrances. Contact Dermatitis 1998: 38: 29-35.

48. Rastogi S C, Heydorn S, Johansen J D, Basketter D A. Fragrance chemicals in domestic and occupational products. Contact Dermatitis 2001: 45: 221-225.

49. Johansen J D, Rastogi S C, Menne T. Exposure to selected fragrance materials. A case study of fragrance-mix-positive eczema patients. Contact Dermatitis 1996: 34: 106-110.

50. Heydorn S, Menne T, Johansen J D. Fragrance allergy and hand eczema - a review. Contact Dermatitis 2003: 48: $59-66$.

51. Heydorn S, Johansen J D, Andersen K E, Bruze M, Svedman C, White I R, Basketter D A, Menne T. Fragrance allergy in patients with hand eczema - a clinical study. Contact Dermatitis 2003: 48: 317-323.

52. Scheinman P L. Prevalence of fragrance allergy. Dermatology 2002: 205: 98-102.

53. Meynadier J M, Meynadier J, Peyron J L, Peyron L. Clinical forms of skin manifestations in allergy to perfumes. Ann Dermatol Venereol 1986: 113: 31-34.

54. Lehucher-Michel M P, Koeppel M C, Lanteaume A, Sayag J. Dyshidrotic eczema and occupation: a descriptive study. Contact Dermatitis 2000: 43: 200-205.

55. Dooms-Goossens A. Cosmetics as causes of allergic contact dermatitis. Cutis 1993: 52: 316-320.

56. Dooms-Goossens A. The red face: contact and photocontact dermatitis. Clin Dermatol 1993: 11: 289-295.
57. Edman B. The influence of shaving method on perfume allergy. Contact Dermatitis 1994: 31: 291-292.

58. de Groot A C, Liem D H. Facial psoriasis caused by contact allergy to linalool and hydroxycitronellal in an after-shave. Contact Dermatitis 1983: 9: 230-232.

59. Goossens A, Merckx L. Allergic contact dermatitis from farnesol in a deodorant. Contact Dermatitis 1997: 37: 179-180.

60. Svedman C, Bruze M, Johansen J D et al. Deodorants: an experimental provocation study with hydroxycitronellal. Contact Dermatitis 2003: 48: 217-223.

61. Johansen J D, Frosch P J, Svedman C, Andersen K E, Bruze M, Pirker C, Menne T. Hydroxyisohexyl 3-cyclohexene carboxaldehyde - known as Lyral: quantitative aspects and risk assessment of an important fragrance allergen. Contact Dermatitis 2003: 48: 310-316.

62. Nardelli A, Taveirne M, Drieghe J, Carbonez A, Degreef H, Goossens A. The relation between the localization of foot dermatitis and the causative allergens in shoes: a 13-year retrospective study. Contact Dermatitis 2005: 53: 201-206.

Address:

An Goossens

Department of Dermatology

University Hospital St Rafaël

Katholieke Universiteit Leuven

Kapucijnenvoer 33

B-3000 Leuven

Belgium

Tel: +3216337860

Fax: + 3216337012

e-mail: an.goossens@uz.kuleuven.ac.be 